\title{
« Les stéréotypes dans la peau » : une approche biopsychosociale des effets situationnels des stéréotypes sur la performance motrice
}

\author{
Aïna Chalabaev* \\ Univ. Grenoble Alpes, Laboratoire SENS, 38041 Grenoble cedex 9, France
}

Reçu le 28 août 2017, Accepté le 11 septembre 2017

\begin{abstract}
Résumé - Des différences d'engagement et de performance entre hommes et femmes sont régulièrement observées dans le domaine de l'activité physique et sportive. Ces différences sont-elles construites socialement, au-delà des facteurs biologiques susceptibles de les expliquer? Les travaux présentés dans cette revue de littérature cherchent à apporter des éléments de réponse à ce questionnement, en se focalisant sur les effets situationnels des stéréotypes, et plus particulièrement sur les processus liés à la menace que l'on peut éprouver lorsque ces stéréotypes sont rendus saillants en contexte évaluatif. Le modèle de la menace du stéréotype est présenté, et les travaux l'ayant testé dans le domaine moteur sont développés. Les résultats montrent que les facteurs sociétaux que constituent les stéréotypes peuvent s'imprimer dans des processus cognitifs, affectifs, motivationnels, physiologiques, et comportementaux, conduisant ainsi à leur confirmation comportementale.
\end{abstract}

Mots clés : stéréotypes, sexe, performance motrice, menace, processus biopsychosociaux

\begin{abstract}
Stereotypes under the skin": a biopsychosocial approach of situational effects of stereotypes on motor performance. Differences in engagement and performance between men and women are regularly observed in the sport and exercise domain. Are these differences socially constructed, beyond the biological factors that are likely to explain them? The studies described in this review aim at providing some answers to this questioning, by focusing on the situational effects of stereotypes, and more particularly on processes related to the threat experienced when these stereotypes are salient in an evaluative context. The stereotype threat model is described as well as the studies that have tested it in the motor domain. Results show that societal factors such as stereotypes can affect cognitive, affective, motivational, physiological, and behavioral processes, leading in turn to their behavioral confirmation.
\end{abstract}

Key words: stereotypes, sex, motor performance, threat, biopsychosocial processes

Imaginez une fille en train d'être évaluée sur une course de sprint lors d'un cours d'éducation physique et sportive, ou une personne âgée évaluée sur un test de force dans un contexte clinique...Qu'est-ce qui détermine leur performance? Bien sûr, leurs compétences techniques et physiques sont importantes, ainsi que leurs habiletés psychologiques à gérer la «pression» et à adopter une motivation et des stratégies optimales au moment du test. Mais si ces facteurs individuels sont déterminants, ils ne sont pas suffisants pour rendre totalement compte de la performance de l'individu. Des facteurs socioculturels plus globaux peuvent également intervenir, même à l'instant précis de l'évaluation, lorsque l'individu est face à luimême. Les filles et les personnes âgées sont notamment

\footnotetext{
*Auteur de correspondance :

aina.chalabaev@univ-grenoble-alpes.fr
}

ciblées par des stéréotypes négatifs dans le domaine physique. Par exemple, ils font l'objet de croyances selon lesquelles les filles courent moins vite que les garçons, et les personnes âgées ont moins de force physique que les jeunes adultes. Ces croyances sont largement partagées dans notre société et peuvent en retour affecter la performance, menant ainsi à leur confirmation comportementale. La présente synthèse se centrera sur les stéréotypes liés au sexe.

Au premier abord, il est commun de penser que ces stéréotypes ont un fond de vérité: des différences physiologiques entre les hommes et les femmes existent, par exemple, en termes de masse musculaire ou de transport de l'oxygène (e.g., Cheuvront, Carter, DeRuisseau, \& Moffatt, 2005), et ces différences expliqueraient pourquoi les femmes courent en moyenne moins vite que les hommes. De ce point de vue, les stéréotypes 
sociaux seraient donc le simple reflet de la réalité. Toutefois, nous proposons ici que les différences de performance physique généralement observées entre les sexes pourraient être en partie construites socialement. Il ne s'agit pas de nier les différences physiologiques existant entre hommes et femmes, mais de proposer que leur performance dans les activités physiques et sportives peut être en partie déterminée par les stéréotypes sociaux, audelà des facteurs individuels d'ordre physiologique, biomécanique, cognitif, affectif, et motivationnel. Cette revue de littérature se focalise sur les effets situationnels des stéréotypes, c'est-à-dire ceux qui se produisent au moment où l'individu réalise une performance, et porte plus précisément sur le phénomène de «menace du stéréotype ».

\section{La menace du stéréotype : définition et paradigme expérimental}

La théorie de la menace du stéréotype (Steele, 1997) vise à comprendre comment la performance de l'individu en contexte évaluatif (e.g., examen) peut être affectée par la présence d'un stéréotype négatif à l'égard d'un groupe auquel il appartient (e.g., les femmes ont de faibles capacités physiques). Plus précisément, la «menace du stéréotype» correspond à la menace ressentie par l'individu lorsqu'il craint d'être jugé sur la base de ce stéréotype plutôt que de sa propre compétence. Cette menace peut le perturber et altérer sa performance, confirmant au final le stéréotype.

Ce phénomène a été pour la première fois mis en évidence par Steele et Aronson (1995), dans le cadre d'un paradigme expérimental qui est devenu classique pour étudier ces effets situationnels des stéréotypes. Ces auteurs ont montré que le simple fait de changer la façon de présenter un test d'intelligence verbale pouvait affecter la performance d'étudiants afro-américains de l'université de Stanford. Lorsque le test était présenté comme une mesure d'intelligence, ces étudiants obtenaient de moins bons résultats que les étudiants européens-américains. Cependant, lorsque le test était présenté comme une tâche de résolution de problèmes sans rapport avec l'intelligence, les participants afro-américains réussissaient aussi bien que les participants européens-américains, après ajustement de leur note obtenue au Scholastic Assessment Test, un test standardisé utilisé comme critère d'admission dans de nombreuses universités nord-américaines. Pour les auteurs, le fait de présenter la tâche comme un test d'intelligence avait activé un stéréotype largement répandu aux États-Unis sur les faibles capacités intellectuelles des afro-américains. Ce contexte aurait généré une pression supplémentaire qui a en retour pesé sur la performance des membres du groupe stéréotypé.

Ce ressenti de menace est supposé concerner principalement les individus qui n'ont pas intériorisé les stéréotypes les concernant, c'est-à-dire ceux qui valorisent le domaine stéréotypé et qui s'y sentent compétents. En effet, selon Steele (1997), l'individu se sent menacé s'il s'identifie au domaine évalué, c'est-à-dire si le regard qu'il porte sur lui-même dépend de sa réussite dans ce domaine. Le fait que les résultats de Steele et Aronson (1995) aient été observés auprès d'étudiants afro-américains ayant réussi à intégrer la très sélective université de Stanford va dans le sens de cette hypothèse. Ainsi, même les individus qui n'ont pas intériorisé les stéréotypes dans le Soi pourraient être confrontés à d'autres obstacles, et voir leurs performances plombées par la menace causée par les stéréotypes. D'après le modèle de Schmader, Johns, et Forbes (2008), l'état de menace du stéréotype est un état de tension générée par une inconsistance entre trois représentations: celle liée au soi, au groupe d'appartenance, et au domaine évalué. Plus précisément, lorsqu'un stéréotype négatif est activé en contexte évaluatif, il entre en conflit avec les attentes de succès de l'individu: d'un côté, il sait que les membres du groupe auquel il s'identifie ont la réputation d'échouer sur la tâche, mais d'un autre côté, lui-même se sent compétent. Ce conflit génère une incertitude quant à sa performance, perturbant en retour cette dernière.

\section{La menace du stéréotype dans les activités physiques et sportives}

Suite à l'étude princeps de Steele et Aronson (1995), plusieurs centaines de travaux ont cherché à mieux comprendre quand et comment l'effet de menace du stéréotype se produit. Ces travaux montrent que ce phénomène concerne différents stéréotypes, associés au sexe (e.g., Spencer, Steele, \& Quinn, 1999), à l'origine ethnique (e.g., Cadinu, Maass, Frigerio, Impagliazzo, \& Latinotti, 2003), au statut socio-économique (e.g., Croizet \& Claire, 1998), à l'âge (e.g., Hess, Auman, Colcombe, \& Rahhal, 2003), ou à l'orientation sexuelle (e.g., Bosson, Haymovitz, \& Pinel, 2004). Ils indiquent également que ce phénomène peut être observé sur différents types de tâches cognitives, comme des tâches de mathématiques (e.g., Rydell, Rydell, \& Boucher, 2010; Spencer, Steele, \& Quinn, 1999), verbales (e.g., Steele \& Aronson, 1995), de mémoire (e.g., Hess, Auman, Colcombe, \& Rahhal, 2003), d'intelligence analytique (e.g., Croizet et al., 2004) ou de rotation mentale (e.g., Wraga, Duncan, Jacobs, Helt, \& Church, 2006). Des effets de menace du stéréotype ont également été rapportés sur des tâches d'interaction sociale (e.g., Bosson, Haymovitz, \& Pinel, 2004), et sur des tâches motrices (e.g., Stone, Lynch, Sjomeling, \& Darley, 1999 ; pour une revue voir Chalabaev \& Sarrazin, 2016).

L'étude de Stone et al. (1999) a été la première à observer ce phénomène dans le domaine moteur. Elle a montré que le fait de présenter une épreuve de golf comme une mesure des capacités physiques (activant un stéréotype négatif à l'égard des européens-américains) diminuait la performance des participants européens-américains par rapport à la condition où le même test était présenté comme une mesure des capacités intellectuelles (activant un stéréotype négatif à l'égard des afro-américains). Les 
résultats inverses ont été observés chez les participants afro-américains. Concernant les stéréotypes négatifs liés au sexe féminin, plusieurs travaux ont depuis observé ce phénomène sur différentes tâches sportives, en golf (Stone \& McWhinnie, 2008), football (Chalabaev, Sarrazin, Stone, \& Cury, 2008; Heidrich \& Chiviacowsky, 2015; Hermann \& Vollmeyer, 2016; Martiny et al., 2015), basket-ball (e.g., Hively \& El-Ayali, 2014; Laurin, 2013, 2017; Martiny et al., 2015), et en tennis (Hively \& El-Alayli, 2014), et sur des tâches motrices de force (e.g., Chalabaev et al., 2013) et de coordination (e.g., Huber, Brown, \& Sternad, 2016; Huber, Seitchik, Brown, Sternad, \& Harkins, 2015). Ces études confirment généralement que la performance diminue suite à l'activation de stéréotypes négatifs à l'encontre des femmes en contexte moteur (sauf Huber et al., 2015, étude 3; Huber et al., 2016) [voir Tab. 1].

\section{Les mécanismes de la menace du stéréotype}

Alors que l'existence du phénomène a été démontrée de façon consistante dans de nombreux travaux, ses processus ont pendant longtemps été peu documentés. Comme le notait Osborne (2001), la menace du stéréotype était un effet sans mécanisme clairement expliqué. Cette difficulté à détecter ces mécanismes peut s'expliquer par la nature autorapportée des mesures utilisées pour les appréhender. Par exemple, les premiers travaux qui ont testé le rôle médiateur de l'anxiété ont souvent utilisé des questionnaires (e.g., Aronson et al., 1999; Steele \& Aronson, 1995; Stone et al., 1999). En contexte sportif, Hermann et Vollmeyer (2016) ont, par exemple, observé une performance plus faible des femmes sur une tâche de football en condition de menace du stéréotype qu'en condition contrôle. Cependant, aucune différence entre les deux groupes n'a été observée sur une mesure auto-rapportée d'inquiétude ressentie. Or, il a été démontré que les individus menacés cherchent à supprimer les émotions négatives qu'ils peuvent ressentir (e.g., Johns, Inzlicht, \& Schmader, 2008), ce qui suggère que les mesures auto-rapportées ne sont pas les plus à mêmes de révéler ce type de processus. À l'heure actuelle, on en sait davantage sur ces processus - notamment dans le cadre des performances cognitives - par l'intermédiaire des travaux qui se sont appuyés sur des mesures non phénoménologiques, telles les mesures cognitives, comportementales et physiologiques. Ces connaissances ont été synthétisées dans le modèle des processus intégrés (integrated process model) de Schmader et al. (2008).

\section{Mécanismes généraux}

\subsection{La motivation à éviter l'échec et l'attention portée à sa propre performance}

Tout d'abord, l'individu peut chercher à résoudre le conflit ressenti en portant son attention sur ses propres comportements. Il a été observé qu'en contexte menaçant, l'individu est plus prudent (e.g., Seibt \& Förster, 2004) et attentif à sa propre performance (e.g., Beilock, Rydell, \& McConnell, 2007). Il semble être également plus vigilant aux signaux d'échec (e.g., Forbes, Schmader, \& Allen, 2008) et à ses propres réactions émotionnelles (e.g., Johns et al., 2008). Cette surveillance accrue de soi-même viserait à diminuer l'incertitude du résultat causée par l'activation des stéréotypes. Elle semble liée au fait que l'individu menacé a tendance à se focaliser sur l'évitement de l'échec.

Cette hypothèse a été testée en articulant la théorie de la menace du stéréotype à celle des buts d'accomplissement (Chalabaev et al., 2008), et plus précisément le modèle tridimensionnel (Elliot \& Church, 1997). Selon ce modèle, lorsqu'ils sont en contexte d'accomplissement (i.e., contexte évaluatif), les individus veulent démontrer leur compétence (ou masquer leur incompétence). En fonction de leur conception de la compétence, cette motivation se traduit par la poursuite de différents buts: des buts centrés sur la maîtrise de la tâche (i.e., buts de maîtrise) lorsqu'ils ont une conception de la compétence en référence à soi, ou des buts centrés sur la comparaison aux autres (i.e., buts de performance) lorsqu'ils ont une conception normative de la compétence. Un apport du modèle tridimensionnel a été d'intégrer le concept de valence motivationnelle (appétive ou aversive) à la théorie des buts, en distinguant les buts de performance-approche (i.e., faire mieux que les autres) des buts de performance-évitement (i.e., éviter de faire moins bien que les autres). Plusieurs travaux ont apporté un soutien empirique à cette distinction, en montrant que les buts de performance-évitement ont des conséquences plus négatives que les buts de performance-approche: ils conduisent notamment à une diminution de la motivation intrinsèque pour la tâche, à l'adoption de conduites d'évitement (e.g., auto-handicap), et in fine, à une diminution de la performance (pour une revue voir Cury, 2004).

Les buts de performance-évitement semblent particulièrement intéressants, car ils sont conceptuellement proches de l'état dans lequel les individus se trouvent quand ils sont menacés par un stéréotype: ils souhaitent éviter d'échouer. L'objectif de l'étude était donc d'examiner si un contexte rendu menaçant par la présence de stéréotypes peut orienter l'individu vers des buts de performance-évitement. Cette hypothèse a été examinée dans le contexte du football, une activité fortement connotée masculine en France (e.g., Plaza, Boiché, Brunel, \& Ruchaud, 2016).

Des analyses de covariance ont tout d'abord montré un effet de l'habillage de la tâche sur la performance postinduction (en contrôlant la performance pré-induction et le niveau du club). Les participantes de la condition contrôle ont effectué le parcours plus rapidement que celles dans la condition menace (capacités physiques), et ont eu tendance à être plus rapides que dans la condition menace (capacités en football). Un effet de l'habillage de la tâche sur l'adoption de buts d'accomplissement a aussi été observé: Les participantes de la condition contrôle ont 


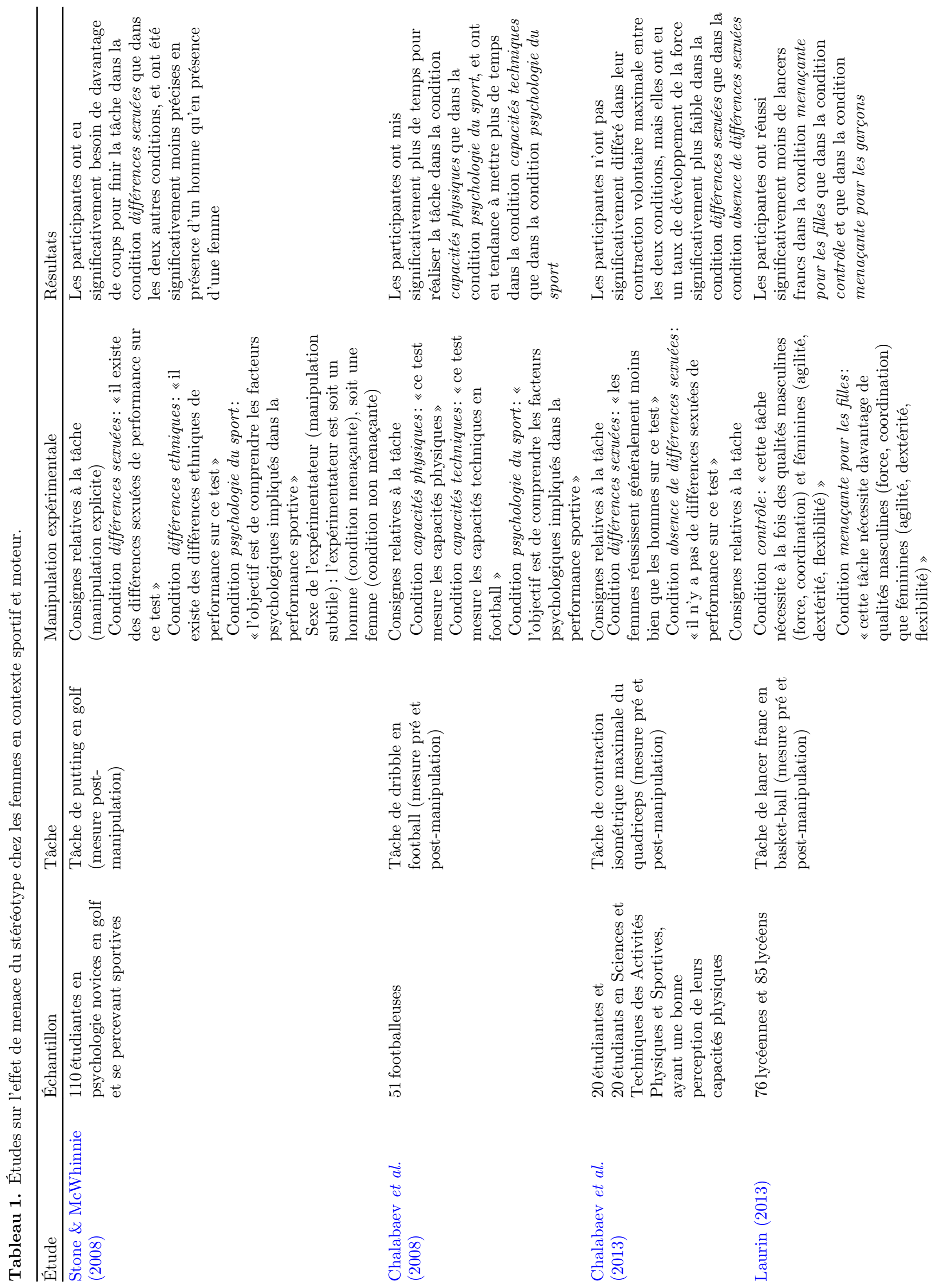




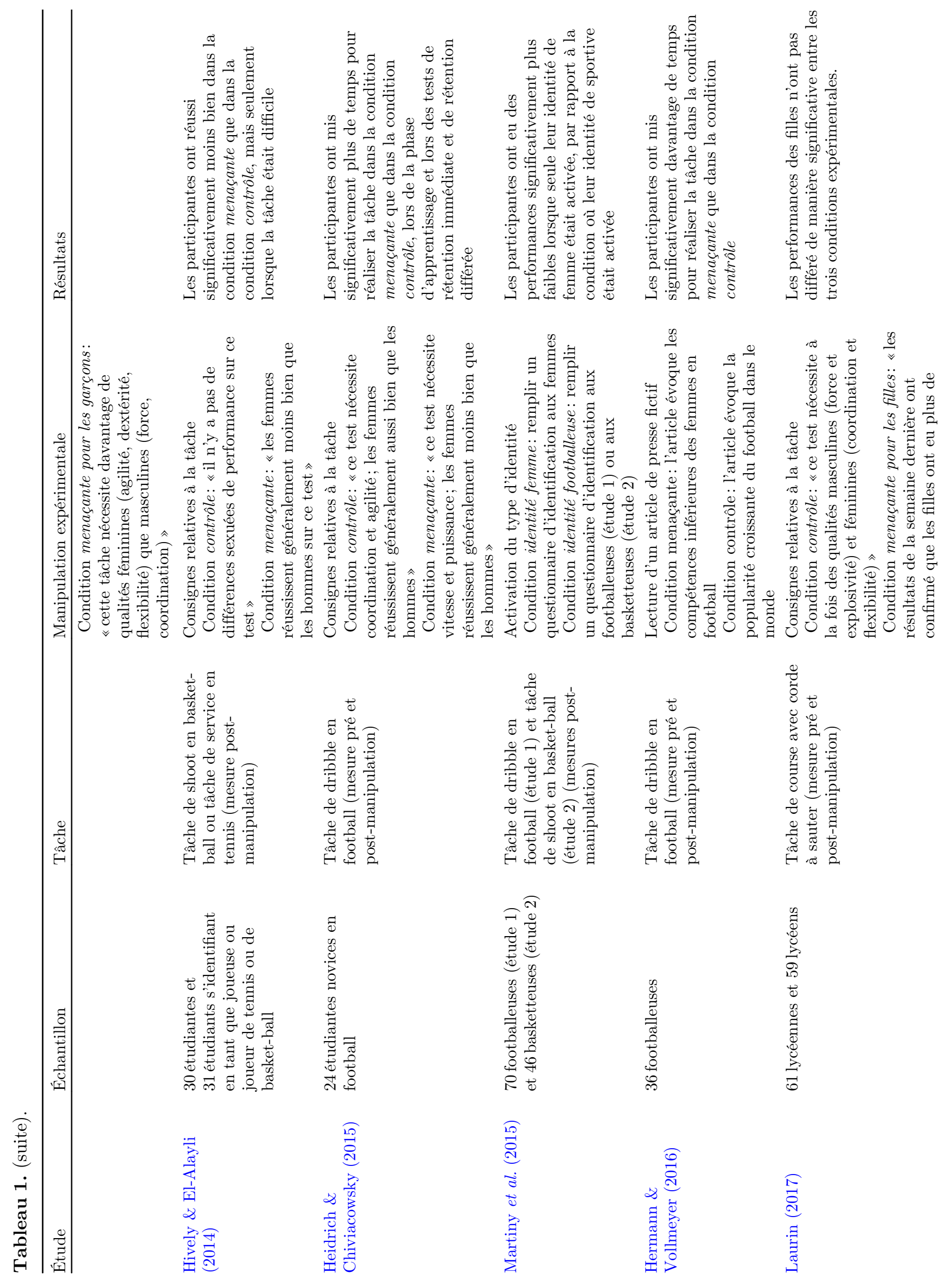




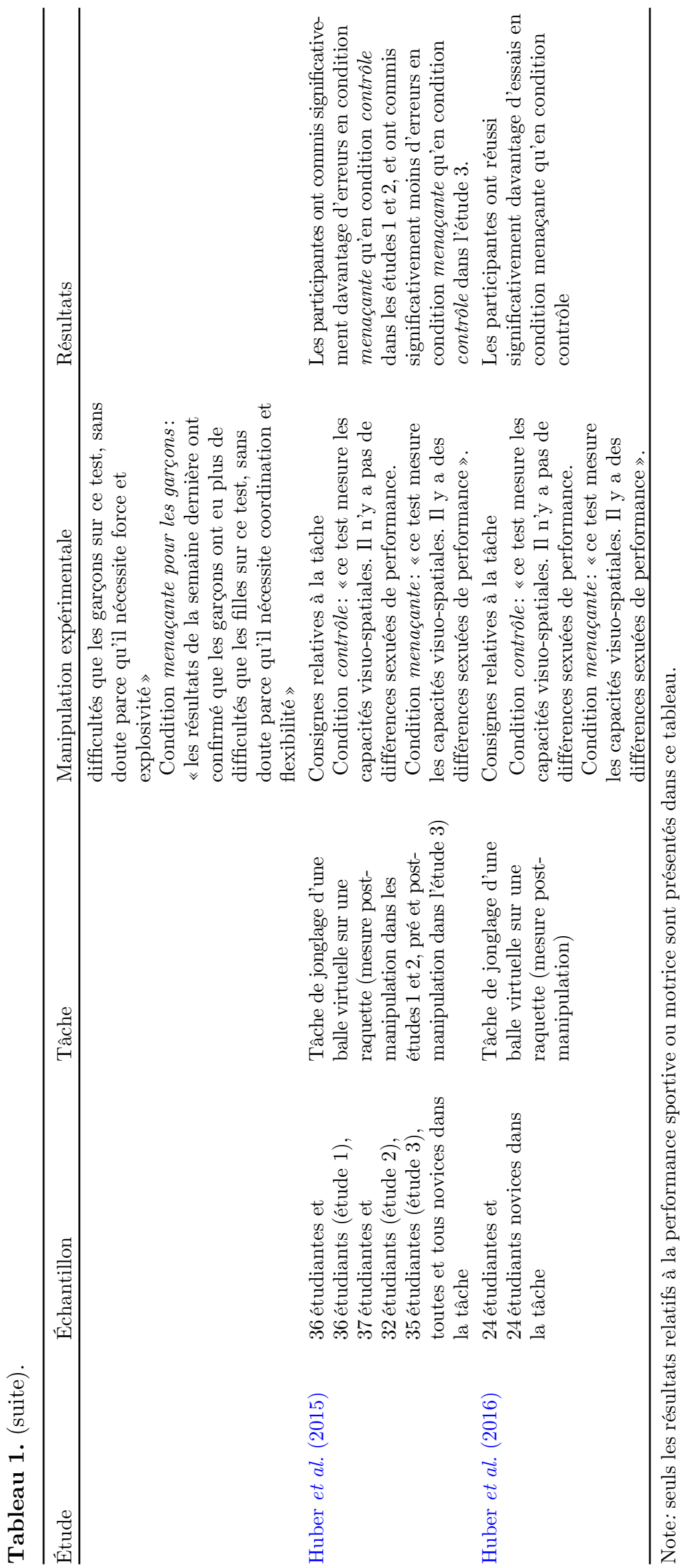


adopté moins de buts performance-évitement (relativement aux buts performance-approche) que dans la condition menace (capacités physiques), et ont eu tendance à moins adopter ces buts que dans la condition menace (capacités en football).

En résumé, les résultats confirment d'une part que le phénomène de menace du stéréotype peut concerner des pratiquantes d'un sport masculin, et donc peu enclines à avoir intériorisé les stéréotypes dans leur concept de soi. D'autre part, ils corroborent l'hypothèse selon laquelle un contexte menaçant peut orienter la motivation de l'individu vers l'évitement de l'échec. Bien que le rôle médiateur de ces buts dans l'effet de l'habillage de la tâche sur la performance n'ait pas été observé dans cette étude, une médiation significative a été rapportée dans une étude subséquente concernant une tâche cognitive (Brodish \& Devine, 2009).

\subsection{Les réponses physiologiques à la menace}

Des réactions de nature physiologique sont également supposées survenir dans une situation de menace du stéréotype. Blascovich, Spencer, Quinn, et Steele (2001) ont été parmi les premiers à les rapporter, en observant une augmentation de la pression artérielle moyenne chez des participants afro-américains lorsqu'ils réalisaient un test cognitif en condition menaçante. D'autres réponses physiologiques à la menace ont depuis été identifiées, telles une diminution de la variabilité de la fréquence cardiaque, indiquant une charge cognitive accrue (Croizet et al., 2004), ou une élévation du taux de cortisol salivaire, reflétant l'activation du système hypothalamo-pituitaireadrénal, qui est généralement impliqué dans les réponses à une menace perçue (Matheson \& Cole, 2004). Des travaux utilisant l'électroencéphalographie (Inzlicht \& Kang, 2010) et l'imagerie par résonance magnétique fonctionnelle (Krendl, Richeson, Kelly, \& Heatherton, 2008) ont montré, par ailleurs, qu'une situation de menace du stéréotype peut activer une région du cortex médio-frontal impliquée dans la régulation émotionnelle: le cortex cingulaire antérieur.

Ces travaux indiquent que le corps répond sur le plan physiologique à la menace ressentie face au stresseur que peuvent représenter des stéréotypes négatifs. Cependant, l'intensité de ces réponses ne donne pas nécessairement d'indication sur leurs retombées pour la performance. En d'autres termes, les réponses au stress ne deviennent pas linéairement maladaptatives en augmentant. En effet, elles peuvent correspondre à l'adaptation normale de l'organisme, qui mobilise de l'énergie afin de faire face au stresseur rencontré. Inversement, une réponse de faible intensité peut indiquer que l'individu se désengage de la tâche, et être dans ce cas maladaptative (Mendes \& Jamieson, 2012). Afin de mieux cerner les conséquences de ces réponses physiologiques sur la performance de l'individu, certains modèles prennent en compte non pas l'intensité de ces réponses, mais la direction motivationnelle qu'elles reflètent. C'est le cas notamment du modèle biopsychosocial de la menace et du défi de Blascovich et de ses collègues (Blascovich \& Mendes, 2000; Blascovich \& Tomaka, 1996). Inspiré des théories de l'évaluation cognitive de Lazarus et Folkman (1984) et de l'endurcissement physiologique de Dienstbier (1989), ce modèle s'applique dans des situations de performance motivée, où l'individu doit agir pour atteindre un objectif important (e.g., passer un examen, faire bonne impression). Dans ces situations, l'individu évalue ses ressources (e.g., compétences) par rapport aux demandes de la tâche (e.g., difficulté, incertitude). S'il estime que ses ressources sont insuffisantes pour faire face à ces demandes, il se trouve dans un état motivationnel de menace, et dans un état de défi dans le cas contraire. L'intérêt du modèle est de proposer des marqueurs physiologiques spécifiques à ces deux états motivationnels. L'état de défi se caractériserait par une activation du système sympatho-adréno-médullaire, qui permet une mobilisation rapide d'énergie. Cette activation est marquée par une élévation de la fréquence cardiaque, de la contractilité ventriculaire (i.e., force contractile du ventricule gauche), et du débit cardiaque (i.e., quantité de sang pompée par le cœur), ainsi que par une vasodilatation des artérioles (i.e., augmentation de leur diamètre), qui permettent in fine d'augmenter le débit sanguin dans le cerveau et la périphérie. L'état de menace se caractériserait également par une activation de ce système, mais celle-ci s'accompagne d'une activation du système hypothalamo-pituitaire-adrénal, qui limite le débit cardiaque et la vasodilatation, et donc le débit sanguin nécessaire pour faire face aux demandes de la tâche.

L'étude de Vick, Seery, Blascovich, \& Weisbuch (2008) est l'une des rares à avoir évalué des réponses physiologiques de menace en contexte rendu menaçant par la présence de stéréotypes. Les résultats ont montré que comparées aux hommes, les femmes étaient davantage dans un état «de menace» lorsque les stéréotypes sur leur infériorité en mathématiques étaient activés. Cependant, aucun effet de l'activation de ces stéréotypes sur la performance à la tâche n'a été rapporté. De façon similaire aux travaux mentionnés précédemment, cette étude indique que l'activation de stéréotypes négatifs peut déclencher des réponses physiologiques à la menace, mais elle ne démontre pas que ces réponses médiatisent l'effet délétère des stéréotypes sur la performance.

Ainsi, même si les mesures physiologiques sont utiles lorsque l'individu ne veut pas ou ne peut pas expliciter ses propres états, elles ne sont pas exemptes de limites. D'une part, la réponse physiologique peut mettre plusieurs minutes avant de se déclencher, notamment si elle met en jeu le système hypothalamo-pituitaire-adrénal, ou au contraire s'interrompre très rapidement si elle implique le système sympatho-adréno-médullaire. D'autre part, lorsque le chercheur ne mesure qu'un seul composant de la réponse au stress (e.g., cortisol), ou lorsqu'il mesure des paramètres qui sont influencés par différents systèmes physiologiques, comme la fréquence cardiaque, qui dépend à la fois du système sympathique et du système parasympathique, cela peut obscurcir les relations entre paramètres physiologiques et performance. Enfin, ces 
relations peuvent être curvilinéaires et n'être donc pas détectées par des analyses de médiation classiques, qui testent des relations linéaires entre médiateur et variable dépendante (Mendes \& Jamieson, 2012).

\subsection{La suppression des pensées et émotions négatives}

L'apparition de pensées et d'émotions négatives constitue le troisième type de réaction en contexte de menace du stéréotype. Il a ainsi été observé que les individus menacés peuvent éprouver de l'anxiété (e.g., Steele \& Aronson, 1995) et avoir des pensées négatives, telles que des doutes (e.g., Stangor, Carr, \& Kiang, 1998) et des inquiétudes quant à leur réussite (e.g., Beilock et al., 2007). Cependant, comme mentionné précédemment, les mesures verbales ont rarement permis de détecter les mécanismes de la menace du stéréotype liés à l'expérience subjective de l'individu (pour une revue voir Wheeler \& Petty, 2001), contrairement aux mesures non verbales. Par exemple, dans une étude de Bosson et al. (2004), les individus stéréotypés ont montré une anxiété comportementale plus élevée en contexte menaçant que dans la condition contrôle, mais n'ont pas rapporté se sentir plus anxieux pour autant.

Pourquoi de telles différences entre mesures verbales et non verbales ont-elles été observées? Une raison possible est que les individus essaient de supprimer les pensées et émotions négatives qu'ils peuvent ressentir en contexte menaçant, car celles-ci sont en conflit avec leur volonté de réussir la tâche. Cette régulation émotionnelle a été observée dans des travaux utilisant une tâche cognitive de détection de sonde, qui se déroule de la façon suivante: suite à l'apparition de deux mots situés au-dessus et audessous du centre de l'écran d'un ordinateur, un point apparaît à l'emplacement d'un des deux mots. Le participant doit indiquer si le point est au-dessus ou audessous du centre de l'écran. Cette tâche est basée sur l'idée que l'anxiété tend à orienter l'attention vers des stimuli menaçants: si les participants identifient plus rapidement la position du point lorsqu'il est situé à l'emplacement d'un mot menaçant (e.g., «peur »), il en est déduit qu'ils sont anxieux. Johns et al. (2008) ont utilisé cette tâche pour étudier les réactions émotionnelles des individus en contexte de menace du stéréotype. Ils ont observé qu'en contexte menaçant, l'attention des individus était orientée prioritairement vers des mots menaçants, confirmant l'hypothèse selon laquelle ce contexte entraîne de l'anxiété. Les auteurs ont alors testé l'idée que les individus essaient de supprimer cette émotion négative, en leur expliquant de quelle façon la tâche permettait de mesurer l'anxiété. Si les individus sont motivés à contrôler leur anxiété, ils devraient éviter de diriger leur attention vers les mots menaçants. Ce sont effectivement les résultats qui ont été observés.

En résumé, d'après le modèle de Schmader et al. (2008), l'incertitude qui naît du conflit contextuel entre des stéréotypes négatifs et les attentes de succès de l'individu entraîne trois types de réponses: une surveillance accrue de sa propre performance, des réactions physiologiques, et des tentatives de suppression de ses pensées et émotions négatives. Paradoxalement, alors que ces réactions visent à résoudre l'incertitude en vue d'obtenir une bonne performance, elles ont à l'inverse des conséquences délétères sur cette dernière. Cependant, les causes de ces effets néfastes diffèrent selon que la tâche est de nature cognitive ou motrice. En effet, les tâches motrices s'appuient sur des processus plus automatisés que les tâches cognitives.

\section{Mécanismes spécifiques aux tâches motrices}

En contexte moteur, alors qu'un novice exécute la tâche en la contrôlant consciemment étape par étape, ce n'est plus le cas lorsque l'individu maîtrise son exécution, celle-ci s'appuyant alors sur des connaissances procéduralisées qui fonctionnent en dehors de la mémoire de travail (e.g., Fitts \& Posner, 1967). Quels sont les mécanismes impliqués? La littérature sur le choking under pressure indique qu'une pression évaluative peut mener les experts à traiter la tâche comme s'ils étaient novices, en surveillant consciemment son exécution étape par étape. Cette surveillance explicite augmente le risque d'erreurs, conduisant l'individu à «craquer » (choke) sous la pression (e.g., Baumeister, 1984; Masters, 1992; pour une revue voir Chalabaev, Sarrazin, \& Heuzé, 2013). Cette littérature suggère qu'une menace causée par un stéréotype pourrait être perturbante parce qu'elle conduit l'individu à porter son attention sur sa réalisation motrice, alors que celle-ci devrait être exécutée de façon automatique. Cette hypothèse a été corroborée dans une étude de Beilock, Jellison, Rydell, McConnell, et Carr (2006). En s'appuyant sur un protocole de double tâche, cette étude a montré que le fait de demander aux individus de mémoriser des mots tout en effectuant une tâche de putting en golf leur a permis de maintenir leur performance à un niveau optimal en contexte menaçant. Selon les auteurs, cette tâche de mémorisation distractive a détourné l'attention des participants de l'exécution de la tâche, permettant à cette dernière d'être réalisée de façon automatique.

Sur la base de ces résultats, nous nous sommes demandés si la menace due aux stéréotypes pouvait affecter la performance motrice sans que ces mécanismes de surveillance explicite n'en soient la cause (Chalabaev et al., 2013). Ce questionnement tirait son origine de résultats montrant qu'un contexte rendu menaçant par la perception de la couleur rouge pouvait diminuer la performance sur une tâche de contraction isométrique maximale du quadriceps (Payen et al., 2011). Or, selon Beilock et Carr (2001), l'exécution de tâches motrices simples n'est pas gênée par les processus de surveillance explicite, car elle ne nécessite pas l'intégration et le séquençage de différentes étapes. Par conséquent, il est peu probable que ces processus soient impliqués dans la diminution de la performance en contexte menaçant observée par Payen et al. (2011). Ce raisonnement est 
renforcé par le fait que cette diminution a concerné la vitesse à laquelle la force était produite dans les 250 premières millisecondes de la contraction, avant que le contrôle attentionnel ne soit possible (e.g., Andersen \& Aagaard, 2006). Si des résultats similaires étaient obtenus dans un paradigme de menace du stéréotype, cela suggèrerait que les processus de surveillance explicite ne sont pas systématiquement impliqués dans le cadre des tâches motrices.

Cette hypothèse a été testée en induisant le stéréotype existant sur les différences de force physique entre hommes et femmes. Les participants devaient réaliser trois contractions isométriques du quadriceps pendant cinq secondes, le plus fort et le plus vite possible. Deux indicateurs de la production de force ont été pris en compte: d'une part, la contraction maximale volontaire (Maximum Voluntary Contraction, MVC), qui correspond au pic de force (Nm) atteint après les 300 premières millisecondes de la contraction (Andersen \& Aagaard, 2006); d'autre part, le taux maximal de développement de la force (Rate of Force Development, RFD) au cours de cette contraction, défini par la pente de la courbe de force exprimée en fonction du temps $(\mathrm{Nm} / \mathrm{sec})$, pour les 10 intervalles suivants : $0-10 \mathrm{~ms}$, 0-20 ms, jusqu'à 0-100 ms. La valeur la plus élevée a été retenue comme mesure du RFD.

Les participants ont ensuite été aléatoirement assignés dans deux conditions expérimentales. Il leur était dit soit que les femmes avaient de mauvaises performances au test par rapport aux hommes (condition stéréotype), soit qu'il n'y avait pas de différence de performance entre les sexes sur cette tâche (condition contrôle). Suite à la manipulation expérimentale, ils ont de nouveau effectué la tâche de force. Les analyses ont révélé un effet d'interaction significatif sur le taux de développement de la force. Des comparaisons deux-à-deux ont indiqué que le RFD des femmes était plus faible dans la condition stéréotype que dans la condition contrôle. Le RFD des hommes n'a quant à lui pas été affecté par la manipulation expérimentale. Concernant la contraction maximale volontaire, les résultats ont également indiqué un effet d'interaction significatif. Mais contrairement aux résultats observés sur le RFD, c'est la performance des hommes qui a été affectée par l'induction de stéréotypes, leur MVC étant plus élevée dans la condition stéréotype que dans la condition contrôle. La MVC des femmes n'a quant à elle pas été affectée par la manipulation expérimentale.

En résumé, ces résultats corroborent l'hypothèse selon laquelle l'activation de stéréotypes négatifs peut altérer la performance motrice sans que des processus de surveillance explicite ne soient impliqués. Dans ce cas, quels pourraient être les mécanismes explicatifs? Étant donné que le RFD est davantage associé à l'étape de planification motrice qu'aux étapes d'exécution et de contrôle de la production de force (e.g., Raghavan, Krakauer, \& Gordon, 2006), il est possible que les pensées et émotions négatives qui apparaissent en contexte menaçant perturbent les processus préparatoires à l'exécution de la tâche. Cette hypothèse reste à tester.

\section{Explications alternatives}

D'autres explications des effets de menace du stéréotype ont été avancées, telle l'hypothèse du « simple effort » (mere effort) [Jamieson \& Harkins, 2007]. Sur la base des travaux sur la facilitation sociale (Zajonc, 1965), ces auteurs ont proposé l'idée que l'induction de stéréotypes négatifs augmenterait la motivation à réussir, ce qui activerait la réponse dominante, c'est-à-dire la réponse la plus fréquente dans une situation donnée (Zajonc, 1965). Or, la réponse dominante est souvent incorrecte sur des tâches difficiles, ce qui expliquerait pourquoi la diminution de performance observée en situation de menace du stéréotype peut ne survenir que sur des tâches difficiles, mais pas sur des tâches faciles (e.g., Hively \& El-Alayli, 2014). Huber et al. (2015) ont ainsi observé un effet de menace du stéréotype sur une tâche de jonglage avec une raquette, mais seulement chez les novices. Lorsque les participantes maîtrisaient la tâche suite à une période d'entraînement, l'induction de stéréotypes négatifs a au contraire conduit à une amélioration de leur performance. Huber et al. (2016) ont par la suite montré que cette induction conduisait à diminuer la variabilité de la performance sur la tâche de jonglage, apportant ainsi un soutien empirique à l'idée que l'induction de stéréotypes négatifs augmenterait la probabilité d'apparition de la réponse dominante.

Wheeler et Petty (2001) ont quant à eux proposé que l'effet de menace du stéréotype sur le comportement serait automatique et n'impliquerait pas de processus motivationnels. Cette hypothèse est basée sur le principe idéomoteur, selon lequel le simple fait de penser à une action augmente la probabilité d'apparition de cette action (James, 1890). Il y aurait donc pour ces auteurs un lien direct entre ce qui est perçu (le stéréotype négatif) et ce qui est fait (la diminution de la performance). La littérature sur les effets d'amorçage des stéréotypes corrobore l'existence de ces effets d'assimilation, même chez des individus non concernés par le stéréotype amorcé (e.g., Bargh, Chen, \& Burrows, 1996; Follenfant, Légal, Dit Dinard, \& Meyer, 2005). Cette hypothèse idéomotrice a été proposée à une époque où les chercheurs éprouvaient des difficultés à identifier les processus motivationnels et émotionnels de la menace du stéréotype. Depuis, les résultats synthétisés dans le modèle de Schmader et al. (2008) apportent un soutien empirique à l'idée que ces processus sont bien impliqués dans le phénomène, et que l'effet de menace du stéréotype est donc différent des effets idéomoteurs des stéréotypes.

\section{Les conditions d'occurrence de la menace du stéréotype}

Un intérêt de cette théorie est qu'elle accorde une place prépondérante au contexte dans lequel les compétences sont évaluées pour expliquer les différences de performance entre groupes sociaux. Steele (1997) reprend les mots de Sartre (1965) en présentant les individus avant 
tout comme des êtres humains dans une situation, ce qui implique que pour les comprendre, il faut d'abord s'interroger sur la situation qui les entoure.

Cette idée est attrayante car elle sous-entend qu'en changeant le contexte évaluatif, il serait possible de réduire les écarts de performance entre groupes sociaux. Cette réduction est généralement observée lorsqu'il est indiqué qu'il n'y a pas de différences entre groupes sur le test que les individus s'apprêtent à passer (e.g., Spencer et al., 1999), ou que le test est présenté comme n'ayant pas de rapport avec la compétence stéréotypée (e.g., Steele \& Aronson, 1995). Cependant, il paraît difficile d'empêcher complètement l'apparition de stimuli menaçants dans le contexte d'évaluation, tant ils sont subtils. Par exemple, le simple fait de devoir indiquer son groupe d'appartenance avant de réaliser le test peut suffire à induire de la menace, tout comme réaliser le test en étant un des seuls représentants de son groupe (pour une revue voir Schmader et al., 2008).

Une approche alternative du problème consiste à identifier les stratégies permettant aux individus de faire face à la menace vécue en contexte évaluatif. Adopter une théorie malléable de la compétence (e.g., Aronson, Fried, \& Good, 2002), mettre en avant un aspect de soi valorisé (e.g., Martiny et al., 2015), ou penser à un membre de son groupe qui est compétent dans le domaine évalué (e.g., Marx, Ko, \& Friedman, 2009) sont des exemples de stratégies qui permettraient de maintenir un niveau optimal de performance en contexte menaçant. Par exemple, Martiny et al. (2015) ont observé qu'en situation de menace du stéréotype, activer une identité positive (être membre d'une équipe de football) permettait d'améliorer la performance de femmes sur une tâche de football.

Dans cette logique, les buts motivationnels peuvent permettre à l'individu de faire face aux effets délétères des stéréotypes, et plus précisément les buts de performanceévitement (Chalabaev, Major, Sarrazin, \& Cury, 2012). Cette hypothèse peut sembler contre-intuitive dans la mesure où la motivation à éviter l'échec, identifiée comme un mécanisme de la menace du stéréotype, est généralement associée à des conséquences négatives (e.g., Elliot, 2005). Cependant, la littérature sur le focus régulateur (regulatory focus, Higgins, 1997, 1998) suggère que ce n'est pas toujours le cas. Ce modèle distingue deux orientations motivationnelles: la promotion, adoptée par l'individu qui cherche à atteindre des choses positives (e.g., gains, succès), et la prévention, adoptée par l'individu qui cherche à éviter des choses négatives (e.g., pertes, échecs). Le but de prévention partage des similitudes conceptuelles avec le but performance-évitement: dans le but de prévention, le succès est atteint lorsque des résultats négatifs sont absents, et dans le but performance-évitement, le succès est atteint lorsque l'échec relativement aux autres est évité.

Or, bien que les premiers travaux sur le focus régulateur considéraient que les buts de prévention étaient maladaptatifs (e.g., Crowe \& Higgins, 1997), il a par la suite été démontré que ces buts peuvent être bénéfiques lorsque les individus sont encouragés à adopter des stratégies qui correspondent à leur orientation motiva- tionnelle (e.g., Keller \& Bless, 2006). En effet, dans cette situation appelée congruence régulatrice (regulatory fit, Higgins, 2000), les individus sont davantage motivés et plus performants. C'est le cas, par exemple, lorsqu'il est demandé à des individus orientés vers la prévention de chercher à éviter une mauvaise performance, car cette stratégie correspond à la sensibilité aux signaux d'échec qui caractérise leur motivation (Keller \& Bless, 2006).

Sur cette base, encourager les individus menacés à adopter un but performance-évitement pourrait leur permettre de maintenir un niveau de performance optimal en générant une situation de congruence régulatrice. Les résultats de Grimm, Markman, Maddox, et Baldwin (2009) vont dans ce sens. Ces auteurs ont manipulé le contexte motivationnel en modifiant le barème de notation d'un test de mathématiques: les participants gagnaient plus de points pour les réponses correctes qu'incorrectes dans la condition de promotion, et perdaient moins de points pour les réponses correctes qu'incorrectes dans la condition de prévention. Conformément aux prédictions de la théorie de la congruence régulatrice, les résultats ont montré que l'effet de menace du stéréotype était supprimé dans la condition de prévention.

Ces résultats ont été récemment corroborés en activant une motivation à l'évitement par des inductions sensorimotrices (Chalabaev, Radel, Masicampo, \& Dru, 2016). Cette étude s'appuie sur les principes de la cognition incarnée, selon lesquels nos pensées, motivations et affects sont incarnés, notamment dans notre système sensorimoteur. Il a ainsi été observé que des mouvements simples peuvent avoir une influence sur des processus cognitifs de haut niveau. Par exemple, contracter un muscle sur le côté gauche du corps déclenche des affects négatifs chez les droitiers (e.g., Bassel \& Schiff, 2001), et inversement, contracter un muscle du côté droit déclenche des affects positifs (e.g., Root, Wong, \& Kinsbourne, 2006). Sur la base de ces résultats, il est possible de faire l'hypothèse que l'induction d'affects négatifs par ce moyen pourrait réduire l'effet de menace du stéréotype. Pour cela, il a été demandé à des participants droitiers d'effectuer une tâche d'arithmétique en contractant une balle en mousse avec leur main droite ou leur main gauche. Les résultats ont confirmé qu'en condition de menace du stéréotype, les participants étaient plus performants lorsqu'ils contractaient leur main gauche que leur main droite. Ces résultats confirment donc l'hypothèse de la congruence régulatrice, cette dernière ayant également été observée dans le cadre d'une tâche de golf (Grimm, Lewis, Todd Maddox, \& Markman, 2016).

\section{Conclusion}

En identifiant le contexte comme un vecteur de l'influence des stéréotypes, les travaux conduits depuis une quinzaine d'années dans le cadre de la menace du stéréotype démontrent que les stéréotypes peuvent se réaliser «ici et maintenant», sans mettre en jeu l'histoire de l'individu, ou en tout cas, au-delà de son histoire personnelle. Elle suggère que même les individus qui n'ont 
pas assimilé les stéréotypes dans leurs perceptions de compétence ou de valeur de l'activité peuvent être négativement affectés par ces derniers. Concernant le domaine physique plus particulièrement, les études sont peu nombreuses mais elles semblent confirmer que les stéréotypes liés au sexe peuvent peser sur la performance des femmes, suggérant que des processus de construction sociale pourraient être impliqués dans les différences entre les sexes observées dans ce domaine, différences pourtant marquées par des facteurs biologiques.

Ceci dit, un débat entoure encore ce phénomène: certains auteurs remettent en cause son existence, en soulignant que l'effet de menace du stéréotype est généralement obtenu dans le cadre de devis expérimentaux, seulement après avoir contrôlé le niveau initial des individus (e.g., Sackett \& Ryan, 2012). Cependant, des effets situationnels des stéréotypes ont été observés en contexte plus écologique (e.g., Danaher \& Crandall, 2008 ; Smeding, Dumas, Loose, \& Régner, 2013), renforçant l'idée qu'ils existent. Deux méta-analyses attestent, par ailleurs, de la réalité du phénomène de menace du stéréotype, montrant en moyenne des tailles d'effet proches $(d=.29$, Walton \& Cohen, $2003 ; d=.26$, Nguyen \& Ryan, 2008). Ces effets varient selon le groupe social ciblé, l'activation implicite ou explicite du stéréotype, et la difficulté de la tâche. Ils peuvent ainsi s'étendre de $d=.17$ à $d=.64$ (Nguyen \& Ryan, 2008).

De plus, parallèlement à la menace du stéréotype, l'activation de stéréotypes en contexte évaluatif peut provoquer un effet de stereotype lift. Ce phénomène renvoie à l'augmentation de la performance du groupe non concerné par le stéréotype (e.g., les hommes lorsqu'un stéréotype négatif envers les femmes est activé). Cette amélioration de la performance résulterait de la comparaison avec un exogroupe (i.e., groupe auquel l'individu n'appartient pas) dévalorisé, comparaison qui améliorerait les attentes de succès de l'individu (Walton \& Cohen, 2003). Comparativement à la menace du stéréotype, très peu d'études ont exploré ce phénomène en contexte moteur (Chalabaev, Stone, Sarrazin, \& Cury, 2008). Celui-ci mériterait pourtant d'être davantage étudié car il peut constituer une source additionnelle d'inégalités entre les sexes suite à l'activation de stéréotypes.

Enfin, des travaux ont récemment montré que les effets des stéréotypes peuvent «déborder» (spillover) des situations évaluatives dans lesquelles ils se produisent. Inzlicht \& Kang (2010) ont observé qu'après avoir été confrontées à des stéréotypes négatifs lors d'un test de mathématiques, les participantes adoptaient des comportements indésirables, comme une alimentation mal équilibrée. Pour ces auteurs, faire face à la menace causée par les stéréotypes nécessite des efforts pour contrôler les émotions négatives ressenties. L'individu a alors moins de ressources pour surmonter les tentations et contrôler ses pulsions. En d'autres termes, les contextes rendus menaçants par la présence de stéréotypes peuvent non seulement affecter la performance de l'individu, mais aussi des comportements qui n'ont qu'un rapport assez lointain avec le domaine stéréotypé.
D'autres «zones d'ombre» subsistent autour de ce phénomène qu'il serait nécessaire d'étudier. Par exemple, est-ce que l'effet de menace du stéréotype perdure sur la tâche ciblée une fois le stéréotype activé? Par ailleurs, si le modèle s'est intéressé en priorité aux individus n'ayant pas intériorisé les stéréotypes les concernant, est-ce que l'effet de menace touche les individus qui ne valorisent pas le domaine stéréotypé? Cette question est particulièrement pertinente en contexte d'éducation physique et sportive, où les élèves ne choisissent le plus souvent pas les activités pratiquées.

\section{Références}

Andersen, L.L., \& Aagaard, P. (2006). Influence of maximal muscle strength and intrinsic muscle contractile properties on contractile rate of force development. European Journal of Applied Physiology, 96, 46-52.

Aronson, J., Lustina, M.J., Good, C., Keough, K., Steele, C.M., \& Brown, J. (1999). When White men can't do math: necessary and sufficient factors in stereotype threat. Journal of Experimental Social Psychology, 35, 29-46.

Aronson, J., Fried, C.B., \& Good, C. (2002). Reducing the effects of stereotype threat on African American college students by shaping theories of intelligence. Journal of Experimental Social Psychology, 38, 113-125.

Bargh, J.A., Chen, M., \& Burrows, L. (1996). Automaticity of social behavior: direct effects of trait construct and stereotype activation on action. Journal of Personality and Social Psychology, 71, 230-244.

Bassel, C., \& Schiff, B.B. (2001). Effects of unilateral facial contractions on approach and withdrawal responses. Neuropsychologia, 39, 282-287.

Baumeister, R.F. (1984). Choking under pressure: self-consciousness and paradoxical effects of incentives on skillful performance. Journal of Personality and Social Psychology, 46, 610-620.

Beilock, S.L., \& Carr, T.H. (2001). On the fragility of skilled performance: what governs choking under pressure? Journal of Experimental Social Psychology, 130, 701-725.

Beilock, S.L., Jellison, W.A., Rydell, R.J., McConnell, A.R., \& Carr, T.H. (2006). On the causal mechanisms of stereotype threat: can skills that don't rely heavily on working memory still be threatened? Personality and Social Psychology Bulletin, 32, 1059-1071.

Beilock, S.L., Rydell, R.J., \& McConnell, A.R. (2007). Stereotype threat and working memory: mechanisms, alleviation, and spillover. Journal of Experimental Psychology: General, 136, 256-276.

Blascovich, J., \& Mendes, W.B. (2000). Challenge and threat appraisals: the role of affective cues. In Forgas J.P. (Ed.), Feeling and thinking: the role of affect in social cognition (pp. 59-82). Paris: Cambridge University Press.

Blascovich, J., \& Tomaka, J. (1996). The biopsychosocial model of arousal regulation. In Zanna M.P. (Ed.), Advances in Experimental Social Psychology (vol.28, pp. 1-51). San Diego: Academic Press.

Blascovich, J., Spencer, S.J., Quinn, D., \& Steele, C. (2001). African Americans and high blood pressure: the role of stereotype threat. Psychological Science, 12, 225-229.

Bosson, J.K., Haymovitz, E.L., \& Pinel, E.C. (2004). When saying and doing diverge: the effects of stereotype threat on self-reported versus non-verbal anxiety. Journal of Experimental Social Psychology, 40, 247-255. 
Brodish, A.B., \& Devine, P.G. (2009). The role of performanceavoidance goals and worry in mediating the relationship between stereotype threat and performance. Journal of Experimental Social Psychology, 45, 180-185.

Cadinu, M., Maass, A., Frigerio, S., Impagliazzo, L., \& Latinotti, S. (2003). Stereotype threat: the effect of expectancy on performance. European Journal of Social Psychology, 33, $267-285$.

Chalabaev, A., \& Sarrazin, P. (2016). Les effets situationnels des stéréotypes. In Fontayne P., \& Chalabaev A. (Eds), Les stéréotypes (pp. 29-44). Paris : Éditions EP\&S.

Chalabaev, A., Sarrazin, P., Stone, J., \& Cury, F. (2008). Do achievement goals mediate stereotype threat?: an investigation on females' soccer performance. Journal of Sport \& Exercise Psychology, 30, 143-158.

Chalabaev, A., Stone, J., Sarrazin, P., \& Croizet, J.-C. (2008). Investigating physiological and self-reported mediators of stereotype lift effects on a motor task. Basic and Applied Social Psychology, 30, 18-26.

Chalabaev, A., Major, B., Sarrazin, P., \& Cury, F. (2012). When avoiding failure improves performance: stereotype threat and the impact of performance goals. Motivation and Emotion, 36, $130-142$.

Chalabaev, A., Sarrazin, P., \& Heuzé, J.-P. (2013). Sport. In Bègue L. \& Desrichard O. (Eds.), Traité de psychologie sociale: la science des interactions humaines (pp. 543-566). Bruxelles: De Boeck.

Chalabaev, A., Sarrazin, P., Fontayne, P., Boiché, J., \& Clément-Guillotin, C. (2013). The influence of sex stereotypes and gender roles on participation and performance in sport and exercise: review and future directions. Psychology of Sport and Exercise, 14, 136-144.

Chalabaev, A., Brisswalter, J., Radel, R., Coombes, S.A., Easthope, C., \& Clément-Guillotin, C. (2013). Can stereotype threat affect motor performance in the absence of explicit monitoring processes? Evidence using a strength task. Journal of Sport \& Exercise Psychology, 35, 211-215.

Chalabaev, A., Radel, R., Masicampo, E.J., \& Dru, V. (2016). Reducing stereotype threat with embodied triggers: a case of sensorimotor-mental congruence. Personality and Social Psychology Bulletin, 42, 1063-1076.

Cheuvront, S.N., Carter, R., DeRuisseau, K.C., \& Moffatt, R.J. (2005). Running performance differences between men and women. Sports Medicine, 35, 1017-1024.

Croizet, J.-C., \& Claire, T. (1998). Extending the concept of stereotype threat to social class: the intellectual underperformance of students from low socioeconomic backgrounds. Personality and Social Psychology Bulletin, 24, 588-594.

Croizet, J.-C., Després, G., Gauzins, M.-E., Huguet, P., Leyens, J.-P., \& Méot, A. (2004). Stereotype threat undermines intellectual performance by triggering a disruptive mental load. Personality and Social Psychology Bulletin, 30, 721-731.

Crowe, E., \& Higgins, E.T. (1997). Regulatory focus and strategic inclinations: promotion and prevention in decision-making. Organizational Behavior and Human Decision Processes, 69, 117-132.

Cury, F. (2004). Évolution conceptuelle de la théorie des buts d'accomplissement dans le domaine du sport. L'Année Psychologique, 104, 295-330.

Danaher, K., \& Crandall, C.S. (2008). Stereotype threat in applied settings re-examined. Journal of Applied Social Psychology, 38, 1639-1655.

Dienstbier, R.A. (1989). Arousal of physiological toughness: implications for physical and mental health. Psychological Review, 96, 84-100.
Elliot, A.J. (2005). A conceptual history of the achievement goal construct. In Elliot A. \& Dweck C. (Eds.), Handbook of competence and motivation (pp. 52-72). New York: Guilford Press.

Elliot, A.J., \& Church, M.A. (1997). A hierarchical model of approach and avoidance achievement motivation. Journal of Personality and Social Psychology, 72, 218-232.

Fitts, P.M., \& Posner, M.T. (1967). Human performance. Belmont: CA, Brooks/Cole.

Follenfant, A., Légal, J.B., Dit Dinard, F.M., \& Meyer, T. (2005). Effet de l'activation de stéréotypes sur le comportement : une application en contexte sportif. Revue Européenne de Psychologie Appliquée, 55, 121-129.

Forbes, C.E., Schmader, T., \& Allen, J.J. (2008). The role of devaluing and discounting in performance monitoring: a neurophysiological study of minorities under threat. Social Cognitive and Affective Neuroscience, 3, 253-261.

Grimm, L.R., Markman, A.B., Maddox, W.T., \& Baldwin, G.C. (2009). Stereotype threat reinterpreted as a regulatory mismatch. Journal of Personality and Social Psychology, 96, 288-304.

Grimm, L.R., Lewis, B., Maddox, W.T., \& Markman, A.B. (2016). Stereotype fit effects for golf putting nonexperts. Sport, Exercise, and Performance Psychology, 5, 39-51.

Heidrich, C., \& Chiviacowsky, S. (2015). Stereotype threat affects the learning of sport motor skills. Psychology of Sport and Exercise, 18, 42-46.

Hermann, J.M., \& Vollmeyer, R. (2016). "Girls should cook, rather than kick!"- Female soccer players under stereotype threat. Psychology of Sport and Exercise, 26, 94-101.

Hess, T.M., Auman, C., Colcombe, S.J., \& Rahhal, T.A. (2003). The impact of stereotype threat on age differences in memory performance. The Journals of Gerontology Series B: Psychological Sciences and Social Sciences, 58, 3-11.

Higgins, E.T. (1997). Beyond pleasure and pain. American Psychologist, 52, 1280-1300.

Higgins, E.T. (1998). Promotion and prevention: regulatory focus as a motivational principle. Advances in Experimental Social Psychology, 46, 1-46.

Higgins, E.T. (2000). Making a good decision: value from fit. American Psychologist, 55, 1217-1230.

Hively, K., \& El-Alayli, A. (2014). "You throw like a girl:" the effect of stereotype threat on women's athletic performance and gender stereotypes. Psychology of Sport and Exercise, 15, 48-55.

Huber, M.E., Brown, A.J., \& Sternad, D. (2016). Girls can play ball: stereotype threat reduces variability in a motor skill. Acta Psychologica, 169, 79-87.

Huber, M.E., Seitchik, A.E., Brown, A.J., Sternad, D., \& Harkins, S.G. (2015). The effect of stereotype threat on performance of a rhythmic motor skill. Journal of Experimental Psychology: Human Perception and Performance, 41, 525-541.

Inzlicht, M., \& Kang, S.K. (2010). Stereotype threat spillover: how coping with threats to social identity affects aggression, eating, decision making, and attention. Journal of personality and social psychology, 99, 467-481.

James, W. (1890). The principles of psychology. New York: Holt.

Jamieson, J.P., \& Harkins, S.G. (2007). Mere effort and stereotype threat performance effects. Journal of Personality and Social Psychology, 93, 544-564.

Johns, M., Inzlicht, M., \& Schmader, T. (2008). Stereotype threat and executive resource depletion: examining the influence of emotion regulation. Journal of Experimental Psychology: General, 137, 691-705. 
Keller, J., \& Bless, H. (2006). Regulatory fit and cognitive performance: the interactive effect of chronic and situational self-regulatory mechanisms on cognitive test performance. European Journal of Social Psychology, 36, 393-405.

Krendl, A.C., Richeson, J.A., Kelley, W.M., \& Heatherton, T.F. (2008). The negative consequences of threat: a functional magnetic resonance imaging investigation of the neural mechanisms underlying women's underperformance in math. Psychological Science, 19, 168-175.

Laurin, R. (2013). Stereotype threat and lift effects in motor task performance: the mediating role of somatic and cognitive anxiety. The Journal of Social Psychology, 15, 687-699.

Laurin, R. (2017). Stereotype threat and lift effects on perceived ability and motor task performance of high school physical education students: the moderating role of stereotype endorsement and domain identification. Movement 85 Sport Sciences, 95, 21-30.

Lazarus, R.S., \& Folkman, S. (1984). The concept of coping. In Monat A. \& Lazarus R.S. (Eds.), Stress and coping: an anthology (3rd ed.) (pp. 189-206). New York: Columbia University Press.

Martiny, S.E., Gleibs, I.H., Parks-Stamm, E.J., MartinyHuenger, T., Froehlich, L., Harter, A.L., \& Roth, J. (2015). Dealing with negative stereotypes in sports: the role of cognitive anxiety when multiple identities are activated in sensorimotor tasks. Journal of Sport \& Exercise Psychology, 37, 379-392.

Marx, D.M., Ko, S.J., \& Friedman, R.A. (2009). The "Obama Effect": how a salient role model reduces race-based performance differences. Journal of Experimental Social Psychology, 45, 953-956.

Masters, R.S.W. (1992). Knowledge, knerves and know how: The role of explicit versus implicit knowledge in the breakdown of a complex sporting motor skill under pressure. British Journal of Psychology, 83, 343-358.

Matheson, K., \& Cole, B.M. (2004). Coping with a threatened group identity: psychosocial and neuroendocrine responses. Journal of Experimental Social Psychology, 40, 777-786.

Mendes, W.B., \& Jamieson, J. (2012). Embodied stereotype threat: exploring brain and body mechanisms underlying performance impairment. In Inzlicht M. \& Schmader T. (Eds.), Stereotype threat: theory, process, and application (pp. 51-68). New York: Oxford University Press.

Nguyen, H.H.D., \& Ryan, A.M. (2008). Does stereotype threat affect test performance of minorities and women? A metaanalysis of experimental evidence. Journal of Applied Psychology, 93, 1314-1334.

Osborne, J.W. (2001). Testing stereotype threat: does anxiety explain race and sex differences in achievement? Contemporary Educational Psychology, 26, 291-310.

Payen, V., Elliot, A.J., Coombes, S.A., Chalabaev, A., Brisswalter, J., \& Cury, F. (2011). Viewing red prior to a strength test inhibits motor output. Neuroscience Letters, 495, 44-48.

Plaza, M., Boiché, J., Brunel, L., \& Ruchaud, F. (2016). Sport $=$ Male $\ldots$ but not all sports: investigating the gender stereotypes of sport activities at the explicit and implicit levels. Sex Roles, 76, 202-217.

Raghavan, P., Krakauer, J.W., \& Gordon, A.M. (2006). Impaired anticipatory control of fingertip forces in patients with a pure motor or sensorimotor lacunar syndrome. Brain, 129, 1415-1425.

Root, J.C., Wong, P.S., \& Kinsbourne, M. (2006). Left hemi-sphere specialization for response to positive emotional expressions: a divided output methodology. Emotion, 6, 47-483.

Rydell, R.J., Rydell, M.T., \& Boucher, K.L. (2010). The effect of negative performance stereotypes on learning. Journal of Personality and Social Psychology, 99, 883-896.

Sackett, P.R., \& Ryan, A.M. (2012). Concerns about generalizing stereotype threat research findings to operational highstakes testing. In Inzlicht M. \& Schmader T. (Eds.), Stereotype threat: theory, process, and application (pp. 249-263). New York: Oxford University Press.

Sartre, J.P. (1965). Anti-Semite and Jew. New York: Schocken Books.

Schmader, T., Johns, M., \& Forbes, C. (2008). An integrated process model of stereotype threat effects on performance. Psychological Review, 115, 336-356.

Seibt, B., \& Förster, J. (2004). Stereotype threat and performance: how self-stereotypes influence processing by inducing regulatory foci. Journal of Personality and Social Psychology, 87, 38-56.

Smeding, A., Dumas, F., Loose, F., \& Régner, I. (2013). Order of administration of math and verbal tests: an ecological intervention to reduce stereotype threat on girls' math performance. Journal of Educational Psychology, 105, 850-860.

Spencer, S.J., Steele, C.M., \& Quinn, D.M. (1999). Stereotype threat and women's math performance. Journal of Experimental Social Psychology, 35, 4-28.

Stangor, C., Carr, C., \& Kiang, L. (1998). Activating stereotypes undermines task performance expectations. Journal of Personality and Social Psychology, 75, 1191-1197.

Steele, C.M. (1997). A threat in the air: how stereotypes shape intellectual identity and performance. American Psychologist, 52, 613-629.

Steele, C.M., \& Aronson, J. (1995). Stereotype threat and the intellectual test performance of African Americans. Journal of Personality and Social Psychology, 69, 797-811.

Stone, J., \& McWhinnie, C. (2008). Evidence that blatant versus subtle stereotype threat cues impact performance through dual processes. Journal of Experimental Social Psychology, $44,445-452$.

Stone, J., Lynch, C.I., Sjomeling, M., \& Darley, J.M. (1999). Stereotype threat effects on Black and White athletic performance. Journal of Personality and Social Psychology, 77, 1213-1227.

Vick, S.B., Seery, M.D., Blascovich, J., \& Weisbuch, M. (2008). The effect of gender stereotype activation on challenge and threat motivational states. Journal of Experimental Social Psychology, 44, 624-630.

Walton, G.M., \& Cohen, G.L. (2003). Stereotype lift. Journal of Experimental Social Psychology, 39, 456-467.

Wheeler, S.C., \& Petty, R.E. (2001). The effects of stereotype activation on behavior: a review of possible mechanisms. Psychological Bulletin, 127, 797-826.

Wraga, M., Duncan, L., Jacobs, E.C., Helt, M., \& Church, J. (2006). Stereotype susceptibility narrows the gender gap in imagined self-rotation performance. Psychonomic Bulletin 8 Review, 13, 813-819.

Zajonc, R.B. (1965). Social facilitation. Science, 149, 269-274.

Citation de l'article : Chalabaev A (2017) «Les stéréotypes dans la peau»: une approche biopsychosociale des effets situationnels des stéréotypes sur la performance motrice. Mov Sport Sci/Sci Mot, 96, 51-63 